\title{
Biodistribution of mesenchymal stem cell-derived extracellular vesicles in a model of acute kidney injury monitored by optical imaging
}

\author{
CRISTINA GRANGE $^{1 *}$, MARTA TAPPARO ${ }^{2 *}$, STEFANIA BRUNO $^{3}$, DEVASIS CHATTERJEE ${ }^{4}$, \\ PETER J. QUESENBERRY ${ }^{4}$, CIRO TETTA $^{5}$ and GIOVANNI CAMUSSI ${ }^{1}$ \\ ${ }^{1}$ Department of Medical Sciences, ${ }^{2}$ Translational Center for Regenerative Medicine and \\ ${ }^{3}$ Department of Molecular Biotechnology and Health Science, University of Torino, Torino, Italy; \\ ${ }^{4}$ Department of Medicine, The Warren Alpert Medical School of Brown University, Providence, RI, USA; \\ ${ }^{5}$ EMEA LA Medical Board, Fresenius Medical Care, Bad Homburg, Germany
}

Received December 5, 2013; Accepted January 17, 2014

DOI: $10.3892 / \mathrm{ijmm} .2014 .1663$

\begin{abstract}
Mesenchymal stem cells (MSCs) contribute to the recovery of tissue injury, providing a paracrine support. Cell-derived extracellular vesicles (EVs), carrying membrane and cytoplasmatic constituents of the cell of origin, have been described as a fundamental mechanism of intercellular communication. We previously demonstrated that EVs derived from human MSCs accelerated recovery following acute kidney injury (AKI) in vivo. The aim of the present study was to investigate the biodistribution and the renal localization of EVs in AKI. For this purpose, two methods for EV labeling suitable for in vivo tracking with optical imaging (OI), were employed using near infrared (NIR) dye (DiD): i) labeled EVs were generated by MSCs pre-incubated with NIR dye and collected from cell supernatants; ii) purified EVs were directly labeled with NIR dye. EVs obtained with these two procedures were injected intravenously (i.v.) into mice with glycerolinduced AKI and into healthy mice to compare the efficacy of the two labeling methods for in vivo detection of EVs at the site of damage. We found that the labeled EVs accumulated specifically in the kidneys of the mice with AKI compared with the healthy controls. After $5 \mathrm{~h}$, the EVs were detectable in whole body images and in dissected kidneys by OI with both types of labeling procedures. The directly labeled EVs showed a higher and brighter fluorescence compared with the labeled EVs produced by cells. The signal generated by the directly labeled EVs was maintained in time, but provided a higher background than that of the labeled EVs produced by cells. The comparison
\end{abstract}

Correspondence to: Dr G. Camussi, Department of Medical Sciences, University of Torino, Corso Dogliotti 14, I-10126 Torino, Italy

E-mail: giovanni.camussi@unito.it

*Contributed equally

Key words: microvesicles, exosomes, mesenchymal stem cells, kidney of the two methods indicated that the latter displayed a greater specificity for the injured kidney.

\section{Introduction}

Cell-derived extracellular vesicles (EVs) contribute to intercellular communication by transferring proteins, bioactive lipids and nucleic acids $(1,2)$. EVs include exosomes released from multivesicular bodies and microvesicles shed from the cell surface. Both vesicle types contain membrane and cytoplasmic constituents of the cells of origin (3). In particular, EVs released from stem cells have been shown to transfer, following receptor-mediated incorporation, into target cells, mRNAs and miRNAs (4-8). Several studies have indicated that the regenerative potential of stem cell-based therapy is related to paracrine/endocrine mechanisms (9-11). EVs play a critical role in transferring regenerative signals from stem cells to the injured tissues (12-14).

Acute kidney injury (AKI) involves the rapid loss of kidney function consequent to a number of causes, which represents one of the main causes of morbidity and mortality in hospitalized patients. Moreover, AKI frequently evolves into chronic renal dysfunction (15). Bone marrow-derived mesenchymal stem cells (MSCs) have been found to improve recovery following AKI induced by toxic agents and ischemia/reperfusion injury (16-18). The observation that MSC-conditioned medium mimics the effect of cell treatment in AKI has suggested a role of MSC-derived factors in coordinating the repair process (11).

Previously, we found that EVs derived from human MSCs accelerated recovery following AKI in SCID mice in a manner comparable to the cells $(13,19,20)$. Moreover, EVs, following incorporation into renal tubular epithelial cells, have been shown to transfer specific mRNA subsets and trigger a regenerative program (13).

To evaluate whether MSC-derived EVs may represent a potential therapeutic tool for AKI, it is essential to investigate in vivo their biodistribution and recruitment within the injured kidneys. Optical imaging (OI) offers the potential for 
a non-invasive study of different targets within the body of living animals. Recently, the OI technique has been improved with the possibility of visualizing a few labeled cells in vivo by using new dyes (21-24). Good candidate dyes to maximize the depth of tissue penetration and reduce the background are near-infrared (NIR) fluorophores (700-900 nm); the absorption coefficient of tissue is very low and light possesses a high potential for penetration (25-27).

The aim of this study was to use OI as a technique to visualize in vivo the biodistribution and localization of EVs derived from MSCs in AKI within $24 \mathrm{~h}$ post-injection (glycerol). For this purpose, we compared two different labeling procedures, one based on direct EV labeling (DL-EV), and the other on the production of labeled EVs by donor cells pre-treated with the dye (LCD-EV).

\section{Materials and methods}

EV isolation. The MSCs were supplied by Lonza (Lonza, Basel, Switzerland) and cultured in the presence of MSC basal medium (MSCBM; Lonza). MSC-derived EVs were collected from the supernatant of MSCs cultured overnight in RPMI-1640 (Lonza) supplemented with $0.5 \%$ of BSA (Sigma-Aldrich, St. Louis, MO, USA). The cell supernatant was centrifuged twice at 3,000 x g for $20 \mathrm{~min}$ to remove cell debris and then ultracentrifuged at 100,000 x g (Beckman Coulter Optima L-90K ultracentrifuge; Beckman Coulter, Brea, CA, USA) for $1 \mathrm{~h}$ at $4^{\circ} \mathrm{C}$. EVs were stored in serum-free RPMI-1640 supplement with $1 \%$ DMSO at $-80^{\circ} \mathrm{C}$. EV protein content was quantified by the Bradford method (Bio-Rad, Hercules, CA, USA).

Labeling procedure. Two labeling protocols were used: i) Cells were stained in suspension and incubated with $5 \mu \mathrm{M}$ Vybrant Cell Tracers DiD [excitation (Ex), 640 nm; emission (Em), 700 nm] or DiI (Ex, 530 nm; Em, 580 nm) (Molecular Probes, Eugene, OR, USA) solution without serum for $20 \mathrm{~min}$ at $37^{\circ} \mathrm{C}$. Cells were then washed in complete medium by centrifugation and cultivated for $24 \mathrm{~h}$ prior to supernatant collection. i) EVs were isolated by ultracentrifugation as previously described (LCD-EVs) (4,13). ii) EVs were directly labeled with $1 \mu \mathrm{M}$ Vybrant Cell Tracers DiI or DiD during the ultracentrifugation procedure (DL-EVs) and then washed twice by ultracentrifugation in $1 \mathrm{X}$ phosphate-buffered saline (PBS) (28).

EV characterization. EVs labeled with the two methods were characterized by cytofluorimetric analysis using FITC- or PE-conjugated antibody against CD44, CD105, CD90 and $\alpha 5$-integrin. FITC or PE mouse non-immune isotypic IgG (Miltenyi Biotec, Bergisch Gladbach, Germany) were used as the controls. Briefly, EVs $(10 \mu \mathrm{g})$ were incubated for $15 \mathrm{~min}$ at $4^{\circ} \mathrm{C}$ with antibodies in $100 \mu \mathrm{l}$ and then diluted in $300 \mu \mathrm{l}$ and immediately acquired. FACS analysis was performed using a guava easyCyte Flow Cytometer (Millipore, Billerica, MA, USA) and analyzed with InCyte software, as previously described $(29,30)$.

The size distribution of the LCD-EVs and DL-EVs was analyzed using a NanoSight LM10 instrument (NanoSight Ltd., Amesbury, UK) equipped with the nanoparticle tracking analyses (NTA) 2.0 analytic software.
In vitro uptake of EVs by human renal tubular epithelial cells. Human renal proximal tubular epitheial cells (PTECs) were labeled following the manufacturer's instructions with the CFSE green dye (Vybrant CFDA SE Cell Tracer kit; Molecular Probes). Cells were incubated for $5 \mathrm{~h}$ at $37^{\circ} \mathrm{C}$ with $50 \mu \mathrm{g} / \mathrm{ml}$ DL-EVs or LCD-EVs and after washing the cells were fixed in $3.5 \%$ paraformaldehyde containing $2 \%$ sucrose. Confocal microscopy analysis was performed using a Zeiss LSM 5 Pascal model confocal microscope (Carl Zeiss, Oberkochen, Germany). Hoechst 33258 dye (Sigma-Aldrich) was added for nuclear staining.

Mouse model of AKI. Studies were conducted in accordance with the national guidelines and regulations and were approved by the Ethics Committee of the University of Torino. Male CD1 nude mice (6-8 weeks old) (Charles River Laboratories, Lyon, France), were fed for 1 week with a special diet (AIN 79; Mucedola, Settimo Milanese, Italy) to reduce tissue autofluorescence. AKI was induced, as previously described (16), by an intramuscular injection of glycerol $(7 \mathrm{ml} / \mathrm{kg}$ body weight of $50 \%$ glycerol solution) into the inferior hind limbs. At 3 days post-injury, the mice were injected intravenously (i.v.) with $200 \mu \mathrm{g}$ of DiD-labeled EVs. Sixteen nude mice with AKI were treated with LCD-EVs and were sacrificed after $5 \mathrm{~h}(\mathrm{n}=9)$ and $24 \mathrm{~h}(\mathrm{n}=7)$. Eleven nude mice with AKI were treated with DL-EVs and were sacrificed after $5 \mathrm{~h}(\mathrm{n}=6)$ and $24 \mathrm{~h}(\mathrm{n}=5)$. The same amount of LCD- and DL-EVs was i.v. injected in 12 and 6 healthy mice, respectively. The animals were sacrificed after $5 \mathrm{~h}$ (LCD-EV, n=6; DL-EV, n=3) and $24 \mathrm{~h}$ (LCD-EV, $\mathrm{n}=6$; DL-EV, $\mathrm{n}=3$ ).

In vitro $O I$. In vitro experiments were performed using the IVIS 200 small animal imaging system (PerkinElmer, Waltham, MA, USA) using the Ex filter at $640 \mathrm{~nm}$ and the Em filter at $700 \mathrm{~nm}$. Background fluorescence was measured and subtracted by setting up a background measurement (Ex filter, $530 \mathrm{~nm}$ ). EV samples were placed in a non-fluorescent black container and the fluorescence intensity of increasing concentrations of LCD-EVs and DL-EVs $(15,30,50$ and $100 \mu \mathrm{g})$ in the same volume was evaluated. Image analysis involved the designation of regions-of-interest (ROI) as the circular area of the well containing the EV concentrations to obtain the average intensity \pm standard deviation (SD), as previously described (31).

In vivo OI. In vivo fluorescence imaging was performed with the same wavelength as described for in vitro acquisition. Identical illumination settings, such as exposure time (2 sec), binning factor (factor of 4), f/stop (set to 2) and 12 fields of view, were used for acquiring all images, and fluorescence Em was normalized to photons per second per centimeter squared per steradian $\left(\mathrm{p} / \mathrm{sec} / \mathrm{cm}^{2} / \mathrm{sr}\right)$. The color image represents the spatial distribution of fluorescence within the animal overlaid on black and white photographs of the mice, collected at the same time. Images were acquired and analyzed using Living Image 4.0 software (PerkinElmer), as previously described (32).

The mice were anesthetized with $2.5 \%$ isoflurane (Merial, Lyon, France) and images were acquired in the prone and supine position after $15 \mathrm{~min}, 5$ and $24 \mathrm{~h}$ post-EV injection. The mice with AKI and the healthy mice treated with PBS were 
used as blank controls for the fluorescence signal of EVs in the AKI and healthy groups, respectively. The fluorescence signal was quantified in the kidney region and in the abdominal area, in ROI draw freehand. The relative mean fluorescence intensity of each ROI was obtained by subtracting the mean fluorescence intensity of the corresponding ROI on the blank mouse from the measured mean fluorescent intensity, as previously described $(22,33)$. Data were expressed as the average radiance $\pm \mathrm{SD}$.

At the end of the experiments (5 or $24 \mathrm{~h}$ post-EV injection), the mice were sacrificed and dissected tissues (kidneys, spleen, liver and lungs) were imaged immediately. The mean fluorescence of each tissue sample was obtained by subtracting the fluorescence intensity of corresponding tissue from the blank mouse, as previously described (33).

Immunofluorescence. Mice were sacrificed at 5 and $24 \mathrm{~h}$ and confocal microscopy analysis (Leica TSC SP5 II) was performed on frozen sections for localization of DiD-labeled EVs in the kidneys. Hoechst 33258 dye (Sigma-Aldrich) was added for nuclear staining. Images were analyzed using ImageJ software.

Statistical analysis. The results are generally expressed as the means \pm SD. Statistical analysis was performed by ANOVA with Dunnet's multi-comparison test or the Newman-Keuls multi-comparison or by the Student's t-test where appropriate. A p-value of $<0.05$ was considered to indicate a statistically significant difference.

\section{Results}

In vitro OI of labeled EVs. The OI of EVs obtained with the two following methods was compared: i) DL-EVs were labeled with DiD after their production and purification; ii) LCD-EVs were obtained by the supernatant of MSCs previously labeled with DiD and then cultured for $24 \mathrm{~h}$ prior to EV collection. OI images were acquired after EV dilution, ranging from 15 to $100 \mu \mathrm{g}$ of $\mathrm{EV}$ proteins, in $100 \mu \mathrm{l}$ of PBS and the average intensity within the entire circle area of each well was calculated. The fluorescence signal correlated linearly with the EV concentration for both labeling methods. DL-EVs were brighter compared with LCD-EVs (Fig. 1).

In vitro characterization of fluorescent EVs and incorporation into renal epithelial tubular cells. EVs labeled with two methods showed the same phenotype as unlabeled EVs. Cytofluorimetric analyses showed their fluorescent signal in the NIR region (Fig. 2A and B) and the presence of several antigens typically expressed by MSCs and by their EVs (13), such as CD44, CD105, CD90 and $\alpha 5$-integrin (Fig. 2C). Regarding size distribution analyzed with NanoSight, LCD-EVs showed a size range of $180 \pm 73 \mathrm{~nm}$ not significantly different from the size distribution of unlabeled EVs $(145 \pm 57 \mathrm{~nm})$. DL-EVs showed a size of $250 \pm 89 \mathrm{~nm}$ with a second peak of larger size probably due to some aggregations occurring during the labeling procedure (Fig. 3A).

To evaluate the ability of labeled EVs to be incorporated by PTECs, $50 \mu \mathrm{g} / \mathrm{ml}$ of EVs labeled with the red dye, DiI, following the same procedure described above, were added to
A

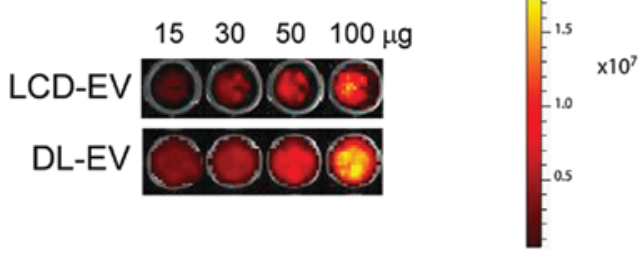

$\left(\mathrm{p} / \mathrm{sec} / \mathrm{cm}^{2} / \mathrm{sr}\right)$

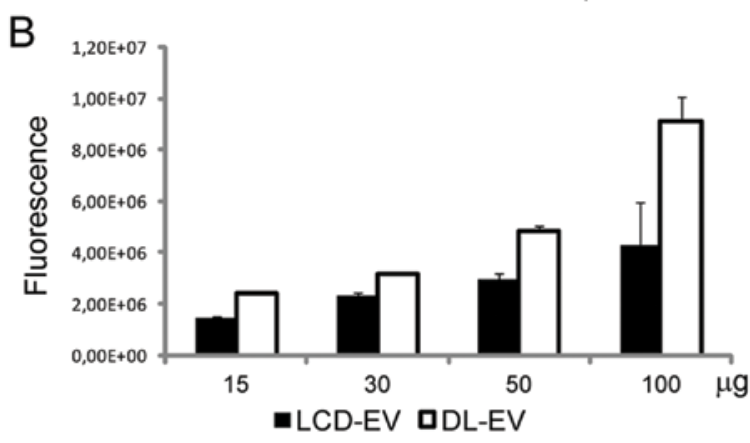

Figure 1. In vitro fluorescence assay. (A) Representative in vitro fluorescence images of extracellular vesicle (EV) dilutions from 15 to $100 \mu \mathrm{g}$ of protein in $100 \mu 1$ of phosphate-buffered saline (PBS). (B) Quantification of fluorescence signal calculated in the entire circle area of each well. Data are expressed as average radiance \pm standard deviation (SD) of three different experiments. LCD-EV, labeled EVs produced by donor cells; DL-EV, directly labeled EVs.

the cells. DL-EVs and LCD-EVs were equally incorporated within PTECs, as observed by confocal microscopy after $5 \mathrm{~h}$ of incubation (Fig. 3B).

In vivo non-invasive OI visualization of $E V$ biodistribution in AKI. The ability of labeled EVs to be visualized by OI on the whole body of live mice was assessed using an IVIS 200 system in a model of AKI induced by an intramuscular glycerol injection, as previously described (13). Three days after the glycerol injection, blood urea nitrogen $(131 \pm 16 \mathrm{mg} / \mathrm{dl})$ and creatinine $(0.9 \pm 0.2 \mathrm{mg} / \mathrm{dl})$ levels were significantly increased compared with the healthy controls $(28 \pm 10$ and $0.2 \pm 0.1 \mathrm{mg} / \mathrm{dl}$, respectively) and were associated with diffuse tubular epithelial injury, characterized by tubular hyaline casts, vacuolization and widespread necrosis of the proximal and distal tubular epithelium, loss of brush border and denudation of the basal membrane (data not shown). The $200 \mu \mathrm{g}$ of DL-EVs or LCD-EVs was inoculated i.v. 3 days following the induction of AKI, when functional and morphological damage had reached its peak (13). Healthy mice were treated with the same amount of EVs in order to evaluate whether the accumulation of EVs was specific for the site of injury. Fig. 4 shows a fluorescent signal in the region of kidneys of AKI mice treated with LCD-EVs and DL-EVs and analyzed posterior after $15 \mathrm{~min}$ and $5 \mathrm{~h}$. However, the intensity of fluorescence in the DL-EVtreated mice with AKI was still present at $24 \mathrm{~h}$ after the i.v. injection and was significantly higher than the fluorescence signal generated by the kidneys of mice with AKI injected with LCD-EVs. In the healthy mice, we observed a fluorescent signal only in the mice treated with DL-EVs after $5 \mathrm{~h}$ in the left dorsal region that may correspond to spleen accumulation; however, the signal decreased rapidly and $24 \mathrm{~h}$ after the i.v. injection was not detectable (Fig. 4). Analyzing the signal 
A

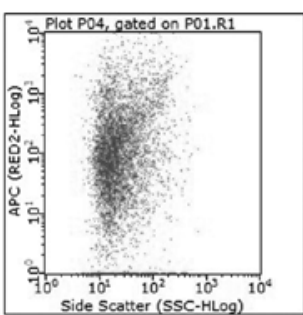

DL-EV

C

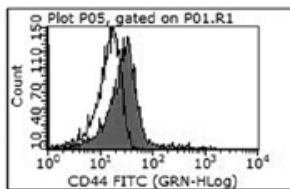

CD44

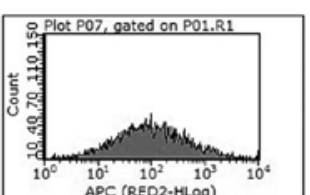

B

LCD-EV


CD90

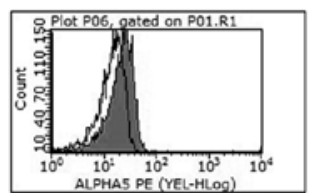

$\alpha 5$-integrin

Figure 2. Evaluation of fluorescence of labeled cell-derived extracellular vesicles (EVs) by a Guava cytofluorimeter. (A and B). Representative dot plots and histogram plots of the fluorescent signal of labeled EVs. (A) DL-EVs show a higher fluorescence compared with (B) LCD-EVs. (C) Representative cytofluorimeter analyses of DL-EVs showing the expression of CD44, CD105, CD90 and $\alpha 5$-integrin. EVs produced with the two staining procedures displayed analogous expression patterns of surface markers (data not shown). White filled histograms indicate the isotypic controls. Three different EV preparations were analyzed with similar results. LCD-EV, labeled EVs produced by donor cells; DL-EV, directly labeled EVs.
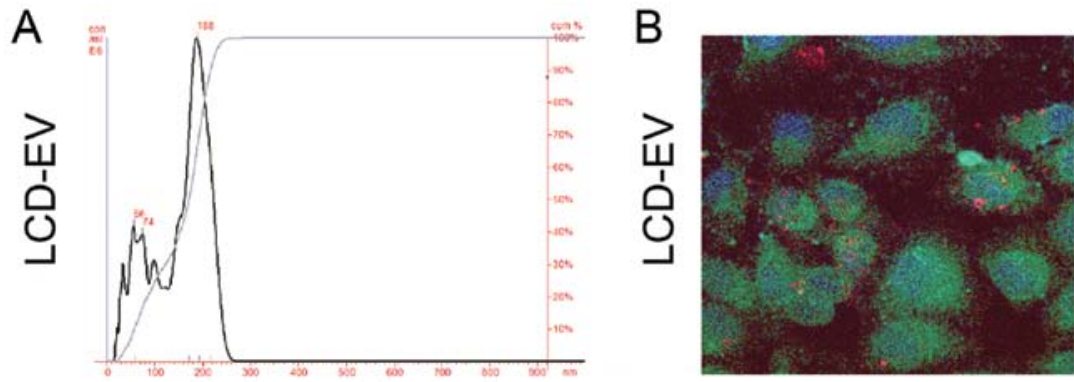

Particle size/concentration

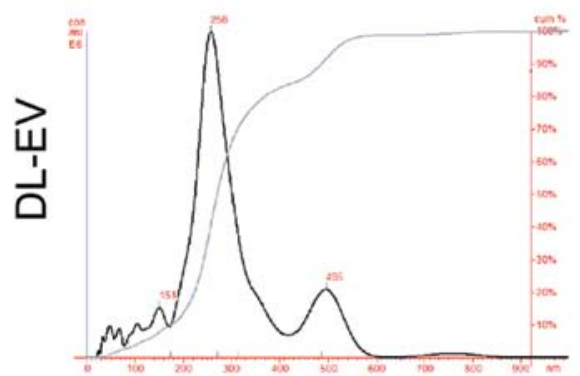

Particle size/concentration

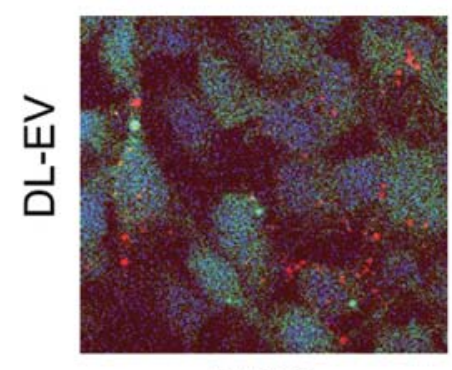

PTEC

Figure 3. Size analysis and incorporation by renal tubular epithelial cells. (A) Representative cell-derived extracellular vesicle (EV) sizes analyzed by measurement with NanoSight. Three different preparation were analyzed with similar results. LCD-EVs maintain the same size distribution than the non-labeled EVs $(180 \pm 73 \mathrm{~nm})$. DL-EVs show a size of $250 \pm 89 \mathrm{~nm}$ with a second larger peak. (B) Representative micrographs of EV incorporation $\left(5 \mathrm{~h}\right.$ at $\left.37^{\circ} \mathrm{C}\right)$ in renal proximal tubular epithelial cells (PTECs). The EVs (red) produced with the two labeling procedures are equally incorporated by PTEC cells (green). Three experiments were performed with similar results. Nuclei were counterstained with DAPI (blue). Original magnification, x630. LCD-EV, labeled EVs produced by donor cells; DL-EV, directly labeled EVs.

in the abdominal area, a higher fluorescence intensity was observed in the AKI groups compared with the healthy groups. Nevertheless, the DL-EV-treated mice with AKI displayed a major increase in fluorescence intensity in comparison with the LCD-EV-treated AKI group, possibly due to the accumulation of EVs in the liver and spleen (Fig. 5).
Ex vivo OI analysis of dissected organs. For each experimental group, the mice were sacrificed at 5 and $24 \mathrm{~h}$ after the EV injection and the fluorescent signal from freshly dissected tissues was quantified immediately by OI. The fluorescence intensity of the kidneys of mice with AKI treated with LCD-EVs and DL-EVs was significantly higher after $5 \mathrm{~h}$ 
A

$15 \mathrm{~min}$
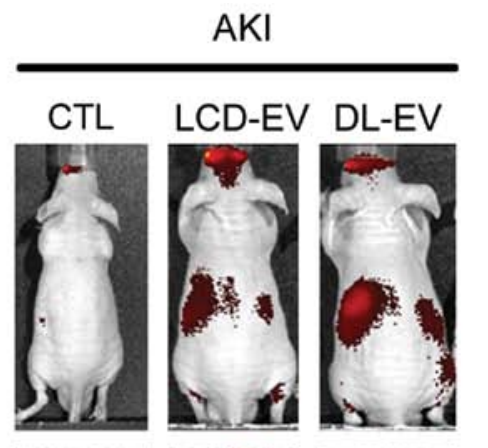

$5 \mathrm{~h}$
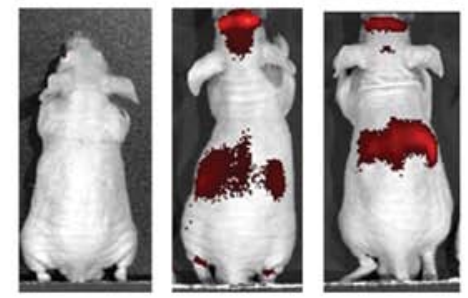

$24 \mathrm{~h}$
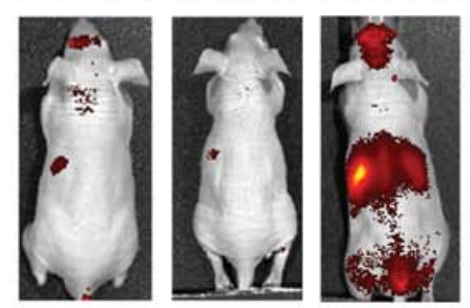

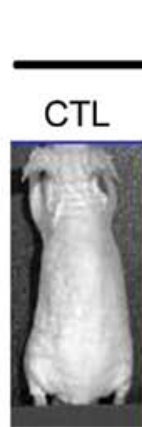

Healthy
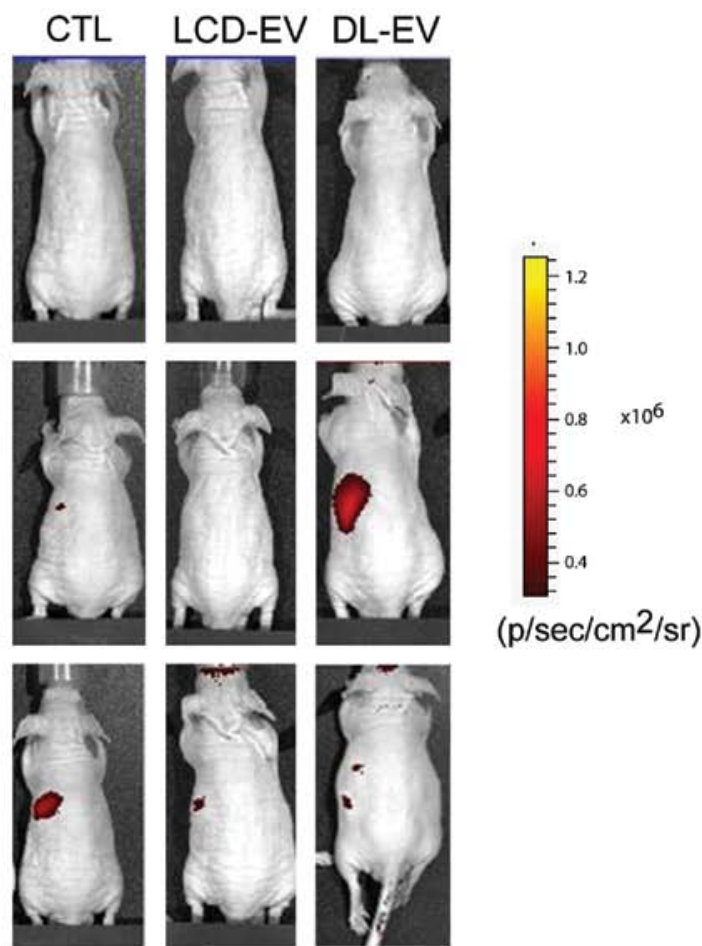

B

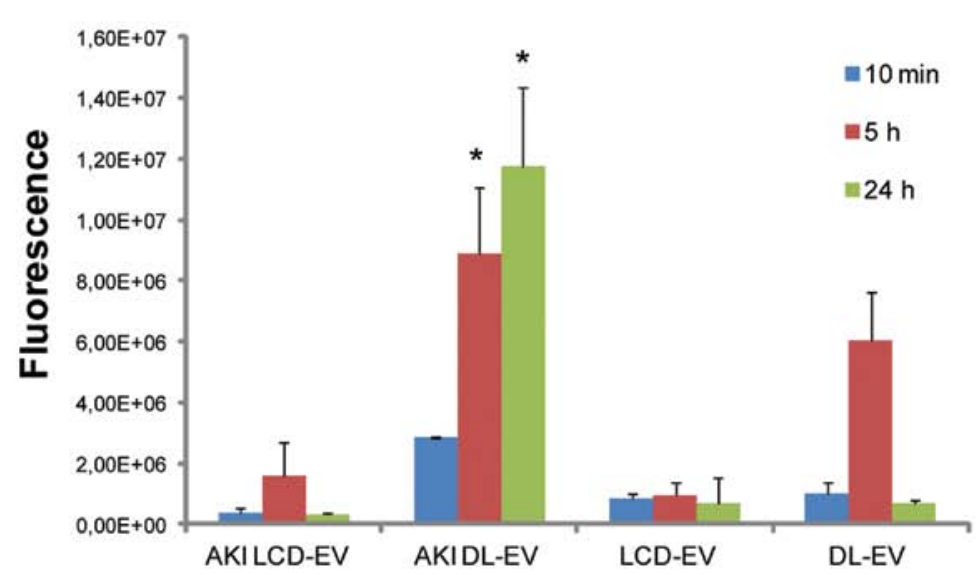

Figure 4. In vivo cell-derived extracellular vesicle (EV) biodistribution in kidney region by optical imaging (OI). (A) Representative OI images, acquired in the posterior position following the induction of acute kidney injury (AKI) in mice and in healthy mice treated intravenously with $200 \mu \mathrm{g}$ of LCD-EVs or DL-EVs or with an equal volume of phosphate-buffered saline (PBS) (CTL). (B) Quantification of fluorescence intensity in regions-of-interest (ROI) draw free hand in the region of kidneys, expressed as the average radiance \pm standard deviation (SD). Sixteen AKI mice were treated with LCD-EVs, 11 AKI mice were treated with DL-EVs; healthy mice received the same amount of LCD- and DL-EVs ( $\mathrm{n}=12$ for LCD-EVs and $\mathrm{n}=6$ for DL-EVs). ANOVA with Newman-Keuls multicomparison test was performed. "p<0.01 AKI DL-EV vs. all the other groups. LCD-EV, labeled EVs produced by donor cells; DL-EV, directly labeled EVs.

compared with the kidneys of mice with AKI treated with PBS [AKI CTL (control)], as shown in Fig 6. Nevertheless, the increase in fluorescence was significantly maintained for $24 \mathrm{~h}$ following treatment in the DL-EV group, whereas it decreased in the LCD-EV group (Fig. 6A). No signal was detected in the kidneys of healthy mice treated with LCD-EVs and DL-EVs, suggesting a specific accumulation of EVs at the site of injury.

The fluorescence signal of DL-EVs was also detected in the spleen and particularly in the liver with high variability. The fluorescence signal of DL-EVs was of low intensity in the lungs of both the AKI and healthy groups. LCD-EVs were detectable only in the injured kidneys.
The presence of LCD-EVs and of DL-EVs within injured kidneys was confirmed by confocal analysis using the appropriate wavelength (Fig. 7). In the kidney sections derived from healthy mice, the presence of fluorescent EVs was almost absent.

\section{Discussion}

The results of the present study demonstrate that it is possible to analyze the biodistribution of EVs either by direct labeling or by the production of labeled EVs from MSCs. In particular, labeled MSC-derived EVs were found to localize within the injured kidneys. 
A

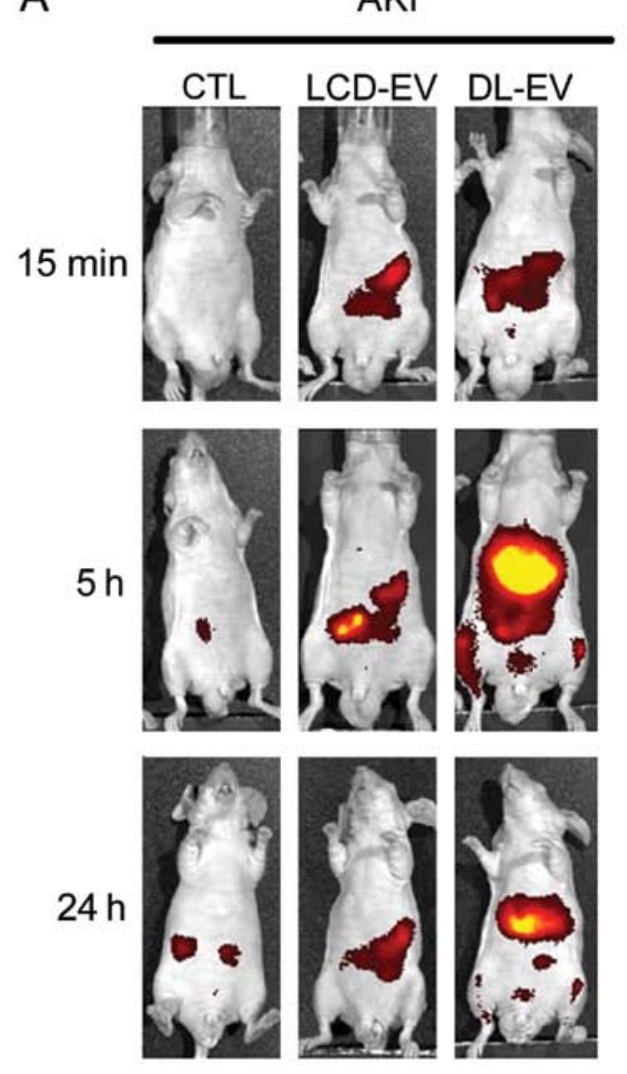

Healthy
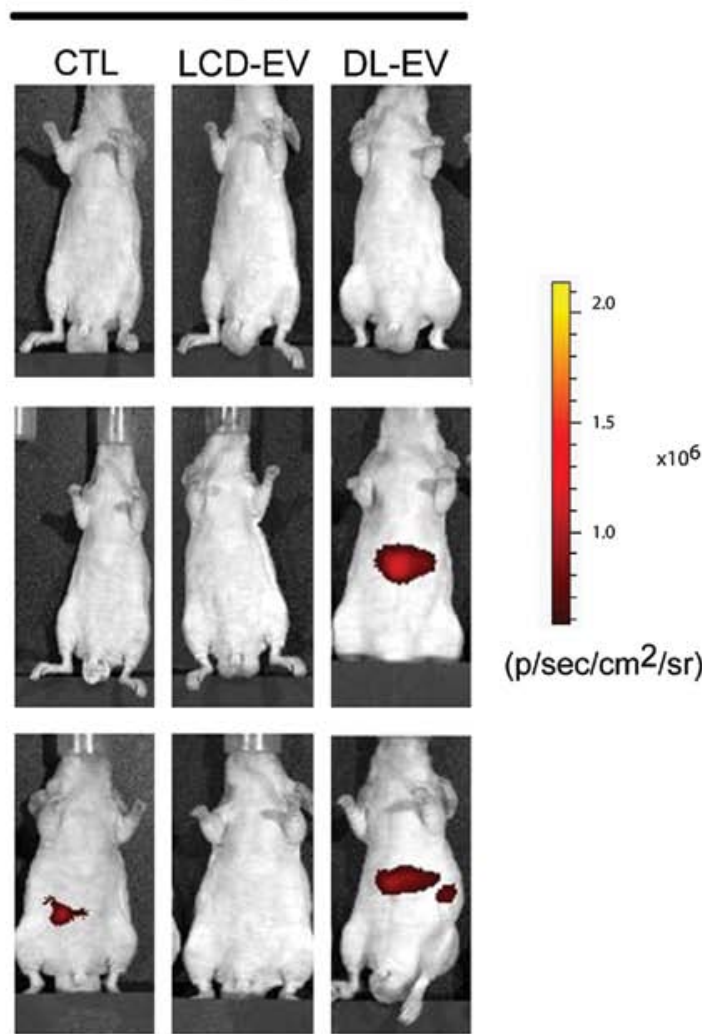

$\times 10^{6}$
B

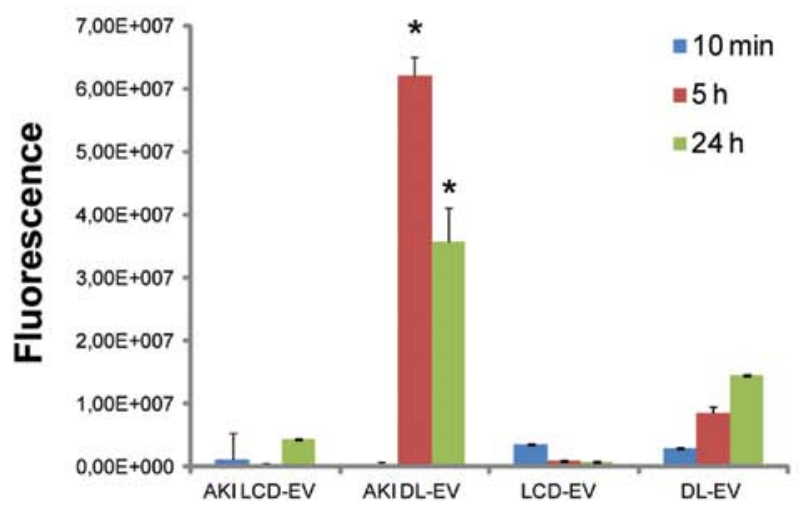

Figure 5. In vivo cell-derived extracellular vesicle (EV) bio-distribution in abdominal area by optical imaging (OI). (A) Representative OI images, acquired the supine position following the induction of acute kidney injury (AKI) in mice and in healthy mice treated intravenously with $200 \mu \mathrm{g}$ of LCD-EVs or DL-EVs or with an equal volume of phosphate-buffered saline (PBS) (CTL). (B) Quantification of fluorescence intensity in regions-of-interest (ROI) draw free hand in the abdominal area, expressed as the average radiance \pm standard deviation (SD). Sixteen AKI mice were treated with LCD-EVs, 11 AKI mice were treated with DL-EVs; healthy mice received the same amount of LCD- and of DL-EVs ( $n=12$ for LCD-EVs and n=6 for DL-EVs). ANOVA with Newman-Keuls multicomparison test was performed. "p<0.01 AKI DL-EV vs. all the other groups. LCD-EV, labeled EVs produced by donor cells; DL-EV, directly labeled EVs.

The imaging of EVs in vivo may contribute to understanding the regenerative potential of EVs released from stem cells. Different approaches to visualize EVs have been proposed, exploiting fluorescent protein-based imaging, such as green fluorescent protein GFP (34), red fluorescent protein (RFP) (35), or the enzymatic activity of the luciferase enzyme which is secreted within exosomes (36). Nevertheless, the use of fluorescent proteins limits the visualization only to the EVs that possess the candidate proteins. Therefore, this technique cannot be applied to all type of vesicles. Since extracellular vesicles are a broad group, differing in content, size and surface markers $(37,38)$ we used a technique that allows the labeling of EV lipid membranes.
The use of small-molecule fluorophores and, in particular, NIR molecules, is a powerful tool to track EVs for noninvasive visualization. These dyes present strong and stable fluorescence in the EV membrane (28). NIR molecules exert a high tissue penetration in concomitance with a low background signal. These dyes have been employed for ex vivo EV detection (28). Since the possibility to trace these dyes in vivo has been shown for labeled cells and antibodies $(33,39)$, in this study, we assessed the possibility to label EVs with DiD for EV tracking.

Previous publications addressing the biodistribution of EVs, have used the ex vivo detection of the dyes in dissected organs (28). Takahashi et al (36), using the luciferase activity 
A
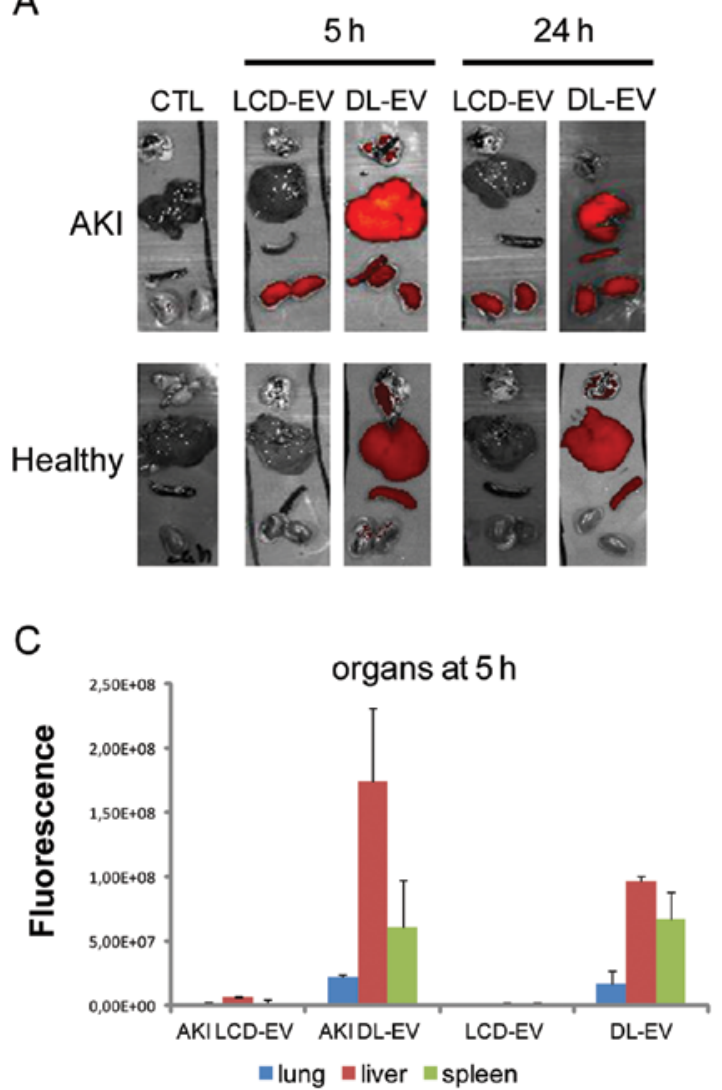

B
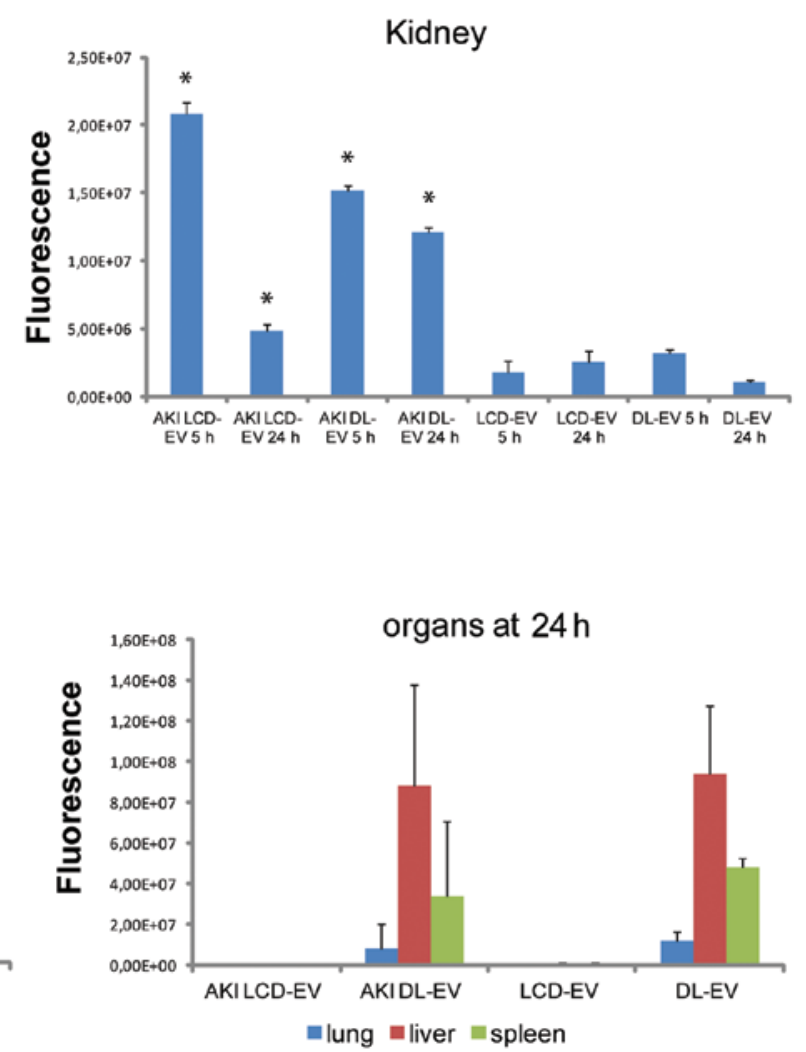

Figure 6. Ex vivo optical imaging (OI) analysis of dissected organs. (A) Representative OI images of dissected organs of mice with acute kidney injury (AKI) and healthy mice treated with LCD- and DL-EVs sacrificed at 5 and $24 \mathrm{~h}$ after injection. (B) Fluorescence quantification of kidneys, expressed as the average radiance \pm standard deviation (SD). ANOVA with Newman-Keuls multi-comparison test was performed. "p<0.05 AKI EVs vs. healthy EVs. (C) Fluorescence quantification, expressed as the average radiance \pm SD, of lungs, liver and spleen. AKI mice treated with LCD-EVs sacrificed at $5 \mathrm{~h}(\mathrm{n}=9)$ and at $24 \mathrm{~h}(\mathrm{n}=7)$; AKI mice treated with DL-EVs sacrificed at $5 \mathrm{~h}(\mathrm{n}=6)$ and at $24 \mathrm{~h}(\mathrm{n}=5)$; healthy mice treated with LCD-EVs sacrificed at $5 \mathrm{~h}(\mathrm{n}=6)$ and at $24 \mathrm{~h}(\mathrm{n}=6)$; healthy mice treated with DL-EVs sacrificed at $5 \mathrm{~h}(\mathrm{n}=3)$ and at $24 \mathrm{~h}(\mathrm{n}=3)$. LCD-EV, labeled EVs produced by donor cells; DL-EV, directly labeled EVs.
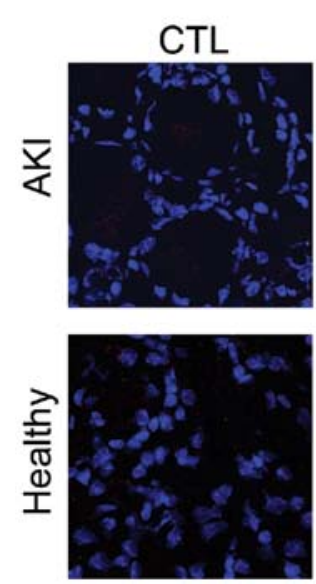

$5 \mathrm{~h}$
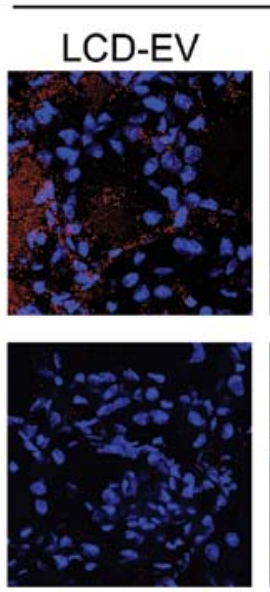

DL-EV
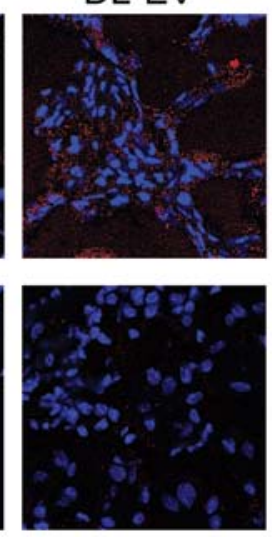

$24 \mathrm{~h}$
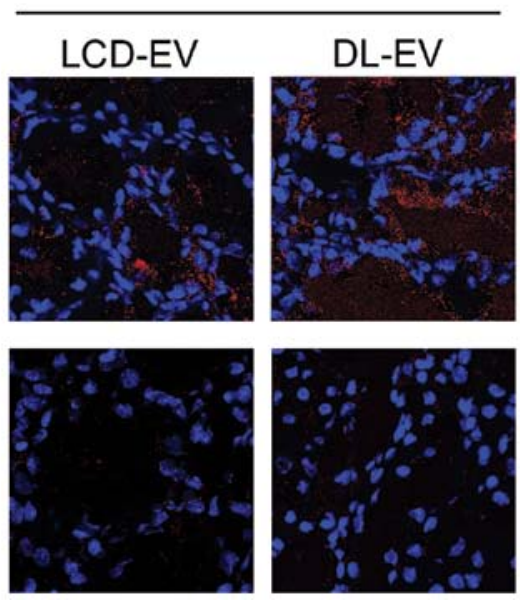

Figure 7. Confocal microscopy of fluorescent cell-derived extracellular vesicles (EVs) in kidneys. Representative micrographs of kidney sections of mice with acute kidney injury (AKI) and healthy mice sacrificed at 5 and $24 \mathrm{~h}$ after EV injection (red). Nuclei were counterstained with Hoechst dye. Two kidney specimens were analyzed for each experimental point. Original magnification, x630. LCD-EV, labeled EVs produced by donor cells; DL-EV, directly labeled EVs.

of EVs, described the possibility to monitor their biodistribution within $4 \mathrm{~h}$.

In this study, we compared the efficiency and sensibility of two labeling methods to visualize EVs in living animals.
DiD-labeled EVs were obtained by direct labeling after their production or from the supernatant of MSCs previously incubated with DiD. Labeled EVs were administered to a mouse model of AKI induced by a glycerol injection and compared 
with healthy controls, to observe their biodistribution. EVs derived from human MSCs have been shown to accelerate the recovery of AKI in different mouse models $(13,19,20)$. In the present study, we found that labeled EVs accumulated specifically in the kidneys of mice with AKI compared with healthy controls. After $5 \mathrm{~h}$, the EVs were detectable in whole body images and in dissected kidneys by OI using both types of labeling procedure. However, the DL-EVs showed a higher and brighter fluorescence compared with the LCD-EVs. In the whole body, the signal generated by the DL-EVs was maintained for $24 \mathrm{~h}$ after the injection, whereas the signal of the LCD-EVs was detectable only in the dissected kidneys. Moreover, the liver and spleen of the mice treated with DL-EVs possessed a fluorescence signal due to a non-specific accumulation of EVs in the excretory organs. Comparing the two methods, the LCD-EVs showed a greater specificity due to their detection only in injured tissue, but the intensity of the fluorescence was lower than that of the DL-EVs. It is known that MSCs are recruited at the site of injury by receptormediated interaction (16). MSC-derived EVs bear the same membrane receptors of MSCs; it is therefore possible that they may accumulate at the site of injury by exploiting the same mechanisms. In addition, the increased permeability in the injured kidney may account for the local accumulation of EVs in AKI.

In conclusion, both these labeling methods were found to be suitable for the in vivo detection of the renal localization of EVs. The localization of EVs in diseased, but not normal kidneys, may explain their beneficial effects on recovery following AKI.

\section{Acknowledgements}

We thank Federica Antico for providing precious technical support. This study was supported by the Stem Kidney grant of Fresenius Medical Care and by the National Center For Advancing Translational Sciences of the National Institutes of Health under Award no. UH2TR000880. The content is solely the responsibility of the authors and does not necessarily represent the official views of the National Institutes of Health.

\section{References}

1. Aliotta JM, Pereira M, Johnson KW, et al: Microvesicle entry into marrow cells mediates tissue-specific changes in mRNA by direct delivery of mRNA and induction of transcription. Exp Hematol 38: 233-245, 2010

2. Ludwig AK and Giebel B: Exosomes: small vesicles participating in intercellular communication. Int J Biochem Cell Biol 44: 11-15, 2012.

3. van Dommelen SM, Vader P, Lakhal S, Kooijmans SA, van Solinge WW, Wood MJ and Schiffelers RM: Microvesicles and exosomes: opportunities for cell-derived membrane vesicles in drug delivery. J Control Release 161: 635-644, 2012.

4. Deregibus MC, Cantaluppi V, Calogero R, Lo Iacono M, Tetta C, Biancone L, Bruno S, et al: Endothelial progenitor cell-derived microvescicles activate an angiogenic program in endothelial cells by a horizontal transfer of mRNA. Blood 110: 2440-2448, 2007.

5. Valadi H, Ekström K, Bossios A, Sjöstrand M, Lee JJ and Lötvall JO: Exosome-mediated transfer of mRNAs and microRNAs is a novel mechanism of genetic exchange between cells. Nat Cell Biol 9: 654-659, 2007.

6. Aliotta JM, Sanchez-Guijo FM, Dooner GJ, et al: Alteration of marrow cell gene expression, protein production, and engraftment into lung by lung-derived microvesicles: a novel mechanism for phenotype modulation. Stem Cells 25: 2245-2256, 2007.
7. Collino F, Deregibus MC, Bruno S, et al: Microvesicles derived from adult human bone marrow and tissue specific mesenchymal stem cells shuttle selected pattern of miRNAs. PLoS One 5: e11803, 2010

8. Ratajczak J, Miekus K, Kucia M, Zhang J, Reca R, Dvorak P and Ratajczak MZ: Embryonic stem cell-derived microvesicles reprogram hematopoietic progenitors: evidence for horizontal transfer of mRNA and protein delivery. Leukemia 20: 847-856, 2006.

9. Jin HJ, Bae YK, Kim M, et al: Comparative analysis of human mesenchymal stem cells from bone marrow, adipose tissue, and umbilical cord blood as sources of cell therapy. Int J Mol Sci 14: 17986-8001, 2013.

10. Humphreys BD and Bonventre JV: Mesenchymal stem cells in acute kidney injury. Annu Rev Med 59: 311-325, 2008.

11. Bi B, Schmitt R, Israilova M, Nishio H and Cantley LG: Stromal cells protect against acute tubular injury via an endocrine effect. J Am Soc Nephrol 18: 2486-2496, 2007.

12. Quesenberry PJ and Aliotta JM: Cellular phenotype switching and microvesicles. Adv Drug Deliv Rev 62: 1141-1148, 2010.

13. Bruno S, Grange C, Deregibus MC, et al: Mesenchymal stem cell-derived microvesicles protect against acute tubular injury. $\mathbf{J}$ Am Soc Nephrol 20: 1053-1067, 2009.

14. Camussi G, Deregibus MC and Cantaluppi V: Role of stemcell-derived microvesicles in the paracrine action of stem cells. Biochem Soc Trans 41: 283-287, 2013.

15. Goldstein SL, Jaber BL, Faubel S, Chawla LS; Acute Kidney Injury Advisory Group of American Society of Nephrology: AKI transition of care: a potential opportunity to detect and prevent CKD. Clin J Am Soc Nephrol 8: 476-483, 2013.

16. Herrera MB, Bussolati B, Bruno S, et al: Exogenous mesenchymal stem cells localize to the kidney by means of CD44 following acute tubular injury. Kidney Int 72: 430-441, 2007.

17. Morigi M, Introna M, Imberti B, et al: Human bone marrow mesenchymal stem cells accelerate recovery of acute renal injury and prolong survival in mice. Stem Cells 26: 2075-2082, 2008.

18. Qi S and Wu D: Bone marrow-derived mesenchymal stem cells protect against cisplatin-induced acute kidney injury in rats by inhibiting cell apoptosis. Int J Mol Med 32: 1262-1272, 2013.

19. Bruno S, Grange C, Collino F, Deregibus MC, Cantaluppi V, Biancone L Tetta C and Camussi G: Microvesicles derived from mesenchymal stem cells enhance survival in a lethal model of acute kidney injury. PLoS One 7: e33115, 2012.

20. Gatti S, Bruno S, Deregibus MC, Sordi A, Cantaluppi V, Tetta C and Camussi G: Microvesicles derived from human adult mesenchymal stem cells protect against ischaemia-reperfusion-induced acute and chronic kidney injury. Nephrol Dial Transplant 26: 1474-1483, 2011.

21. Boddington S, Henning TD, Sutton EJ and Daldrup-Link HE: Labeling stem cells with fluorescent dyes for non-invasive detection with optical imaging. J Vis Exp 14: pii686, 2008.

22. Tögel F, Yang Y, Zhang P, Hu Z, and Westenfelder C: Bioluminescence imaging to monitor the in vivo distribution of administered mesenchymal stem cells in acute kidney injury. Am J Physiol Renal Physiol 295: F315-F321, 2008.

23. Sutton EJ, Henning TD, Boddington S, Demos S, Krug C, Meier R, Kornak J, et al: In vivo magnetic resonance imaging and optical imaging comparison of viable and nonviable mesenchymal stem cells with a bifunctional label. Mol Imaging 9: 278-290, 2010.

24. Garrovo C, Bergamin N, Bates D, et al: In vivo tracking of murine adipose tissue-derived multipotent adult stem cells and ex vivo cross-validation. Int J Mol Imaging 2013: 426961, 2013.

25. Chatterjee DK, Gnanasammandhan MK and Zhang Y: Small upconverting fluorescent nanoparticles for biomedical applications. Small 6: 2781-2795, 2010.

26. Rao J, Dragulescu-Andrasi A and Yao H: Fluorescence imaging in vivo: recent advances. Curr Opin Biotechnol 18: 17-25, 2007.

27. Boddington SE, Sutton EJ, Henning TD, Nedopil AJ, Sennino B, Kim A and Daldrup-Link HE: Labeling human mesenchymal stem cells with fluorescent contrast agents: the biological impact. Mol Imaging Biol 13: 3-9, 2011.

28. Hood JL, San RS and Wickline SA: Exosomes released by melanoma cells prepare sentinel lymph nodes for tumor metastasis. Cancer Res 71: 3792-3801, 2011.

29. Ansa-Addo EA, Lange S, Stratton D, et al: Human plasma membrane-derived vesicles halt proliferation and induce differentiation of THP-1 acute monocytic leukemia cells. J Immunol 185: 5236-5246, 2010. 
30. Grant R, Ansa-Addo E, Stratton D, et al: A filtration-based protocol to isolate human plasma membrane-derived vesicles and exosomes from blood plasma. J Immunol Methods 371: 143-151, 2011.

31. Sutton EJ, Boddington SE, Nedopil AJ, Henning TD, Demos SG, Baehner R, Sennino B, et al: An optical imaging method to monitor stem cell migration in a model of immune-mediate arthritis. Opt Express 17: 24403-24413, 2009.

32. Herrera MB, Fonsato V, Bruno S, et al: Human liver stem cells improve liver injury in a model of fulminant liver failure. Hepatology 57: 311-319, 2013.

33. Zou P, Xu S, Povoski SP, et al: Near-infrared fluorescence labeled anti-TAG-72 monoclonal antibodies for tumor imaging in colorectal cancer xenograft mice. Mol Pharm 6: 428-440, 2009.

34. Lai CP, Tannous BA and Breakefield XO: Noninvasive in vivo monitoring of extracellular vesicles. Methods Mol Biol 1098: $249-258,2014$.
35. Suetsugu A, Honma K, Saji S, Moriwaki H, Ochiya T and Hoffman RM: Imaging exosome transfer from breast cancer cells to stroma at metastatic sites in orthotopic nude-mouse models. Adv Drug Deliv Rev 65: 383-390, 2013.

36. Takahashi Y, Nishikawa M, Shinotsuka H, Matsui Y, Ohara S, Imai T, Takakura Y: Visualization and in vivo tracking of the exosomes of murine melanoma B16-BL6 cells in mice after intravenous injection. J Biotechnol 165: 77-84, 2013.

37. Raposo G and Stoorvogel W: Extracellular vesicles: exosomes, microvesicles, and friends. J Cell Biol 200: 373-383, 2013.

38. Camussi G, Deregibus MC, Bruno S, Cantaluppi V and Biancone L: Exosomes/microvesicles as a mechanism of cell-tocell communication. Kidney Int 78: 838-848, 2010.

39. Kalchenko V, Shivtiel S, Malina V, Lapid K, Haramati S, Lapidot T, Brill A and Harmelin A: Use of lipophilic nearinfrared dye in whole-body optical imaging of hematopoietic cell homing. J Biomed Opt 11: 050507, 2006. 